\title{
Integración de sistemas de información para la innovación en la gestión operativa de unidades productivas agrícolas. Un caso de estudio en Colombia
}

\author{
Helga Patricia Bermeo Andrade ${ }^{a}$, Dora Luz González-Bañales ${ }^{b}$ \\ ${ }^{a}$ Grupo GINNOVA, Universidad de Ibagué, Colombia, helga.bermeo@unibague.edu.co \\ ${ }^{\mathrm{b}}$ Departamento de Sistemas y Computación, Instituto Tecnológico de Durango/Tecnológico Nacional \\ de México,doraglez@itdurango.edu.mx
}

\begin{abstract}
Resumen
En esta ponencia se presentan los resultados del Desarrollo, transferencia y evaluación de un Sistema de Información de Gestión de Fincas (SIGF o FMIS por sus siglas en inglés Farm Management Information Systems) en su versión web, a un colectivo de productores del sector hortofrutícola en Colombia. El proceso de desarrollo se sustenta en la transferencia del SIGF y la evaluación de experiencia de usuario. Los resultados obtenidos evidencian dos logros en particular: el primero relacionado con el desarrollo de una herramienta innovadora que transforma la gestión operativa de unidades productivas agrícolas, en lo que corresponde a la planificación, programación, ejecución y control de tareas, y el segundo con la aplicación de un mecanismo para facilitar el involucramiento de usuarios con experiencia en la actividad agrícola en el desarrollo de un SIGF.
\end{abstract}

Palabras clave: Sistemas de Información para la Gestión de Fincas, Experiencia de Usuario, Transferencia de Tecnología, Innovación en Procesos, Gestión Operativa Agrícola

\section{Introducción}

En la actual sociedad de la información y el conocimiento, la integración de las Tecnologías de Información y Comunicación (TIC) hacen parte de la estrategia para apoyar la gestión eficiente y moderna de unidades de producción agrícola, así como para la generación de sistemas sostenibles de alimentación (Bilali \& Allahyari, 2018). Dentro de dicha integración, se encuentran los denominados Sistemas de Información para la Gestión de Fincas (entendiendo como finca como unidad productiva agrícola) (SIGF), los cuales han surgido como apoyo para la gestión de cultivos y para el manejo administrativo y financiero 
de las fincas (Tummers et al., 2019). Con base en lo anterior, un administrador agrícola se enfrenta al hecho de que no basta su experiencia como agricultor para lograr la sostenibilidad y el éxito comercial, ahora necesita desarrollar capacidades para estar al tanto de los últimos avances en investigación y tecnología agrícola, y tener habilidades para la toma de decisiones soportado en el uso de las TIC (Fountas et al., 2015; Kaloxylos et al., 2012).

Asimismo, la incorporación de las TIC en la gestión de fincas se ha convertido en una cuestión particularmente relevante y necesaria para los productores de países con gran tradición agrícola, para ganar productividad y ser competitivos en esta actividad productiva (Pradhan et al., 2018). No obstante, la integración de este tipo de tecnologías en países en desarrollo como Colombia, se ve limitada por la deficiente infraestructura tecnológica presente en las unidades productivas y en las zonas rurales, y por poca integración logística efectiva entre los actores que conforman estas agro-cadenas de suministro (OCDE, 2015).

En lo que respecta a los SIFG, la tendencia es que éstos operen a través de Internet (Fountas et al., 2015; Kaloxylos et al., 2012), de tal manera que puedan ser orientados a incorporar un conjunto de sistemas planificados para recolectar, procesar, almacenar y diseminar datos en la forma necesaria para llevar a cabo las operaciones y funciones de una finca. Con base en lo anterior se observa que estos sistemas han evolucionado de simples registros de fincas hasta sistemas sofisticados y complejos para respaldar la gestión de la producción, cuyo propósito es satisfacer las crecientes demandas para reducir los costos de producción, cumplir con los estándares agrícolas y mantener una alta calidad y seguridad del product (Fountas et al., 2015).

Abordando el desarrollo de un SIGF en el contexto del sector agrícola Colombiano, el trabajo que aquí se presenta describe la experiencia de desarrollo, transferencia y evaluación del SIGF denominado itagüe ${ }^{\circledR}$ (http://itague.co), el cual fue desarrollado para motivar la innovación en el proceso de la gestión operativa de unidades productivas agrícolas productoras de fruta en la región central del Tolima (Colombia), en lo que corresponde a las labores de planificación, programación, ejecución y control de tareas en finca. itagüe ${ }^{\circledR}$ representa un apoyo a la innovación en proceso en el sector analizado, toda vez que la incorporación del mismo representó para los productores intervenidos, una metodología nueva frente a su quehacer productivo convencional, logrando involucrarlos en la fase de desarrollo, transferencia y evaluación del sistema itagüe $\AA$. 


\section{Metodología}

Las metodologías utilizadas para las fases de desarrollo, transferencia y evaluación del SIGF itagüe $\circledR$, se distinguen según su propósito en los siguientes apartados.

\subsection{Fase de desarrollo}

En su fase de desarrollo, el SIGF diseñado se alineó a las metodologías propias del diseño centrado en el usuario, como la propuesta en el año 2010 en la norma ISO 9244-210, para asegurar que éste satisficiera los requisitos de una eficiente gestión operativa en fincas (Figura 1).

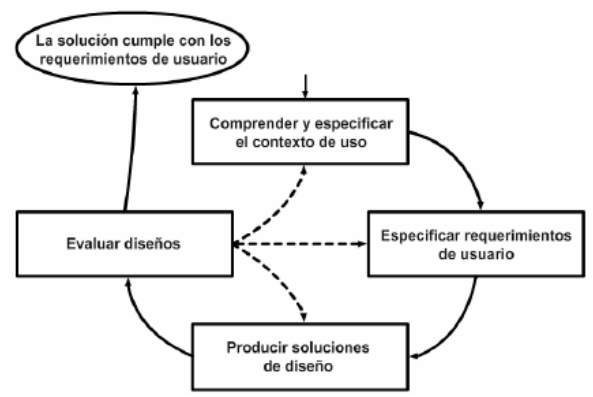

Fig. 1. Proceso iterativo del Diseño Centrado en el Usuario según la norma ISO

Fuente: (Sánchez, D., Ibarra, J., Flores, B., López, 2012)

\subsection{Fase de transferencia}

La etapa de transferencia del SIGF se apoyó en la metodología propuesta por González (Gonzalez, 2011), como se presentan en la Figura 2, para a su vez asegurar que la herramienta fuera apropiada convenientemente por cada uno de los usuarios de la misma (dueños/administradores de fincas, operarios de fincas, asesores técnicos de fincas.

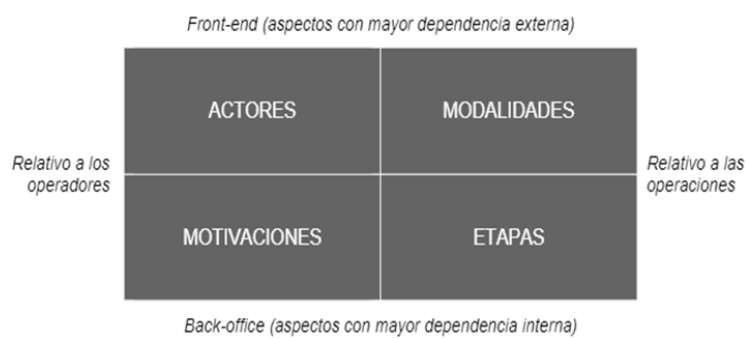

Fig. 2. Elementos que conforman el proceso de transferencia de tecnología Fuente: (Gonzalez, 2011) 
Integración de sistemas de información para la innovación en la gestión operativa de unidades productivas agrícolas. Un caso de estudio en Colombia

\subsection{Fase de pruebas}

Las pruebas con usuarios de itagüe ${ }^{\circledR}$ se realizaron con la participación de 15 productores agrícolas vinculados al sector de producción de mango de la zona centro del Tolima (Colombia), propietarios de unidades productivas agrícolas que oscilaron entre 10 y 50 hectáreas de cultivo permanente. Del total de participantes, el 93\% (14) eran productores dueños de las fincas. El 58\% (7) de ellos tenía menos de 55 años, y el resto tenía 56 años o más (42\%). El 80\% (12) hombres.

Para evaluar la experiencia de usuario del SIGF itagüe ${ }^{\circledR}$ en la fase de pruebas, se utilizó una adaptación del cuestionario USE desarrollado por Lund (2001), cuyo trabajo cuenta a su vez con bases desde los trabajos de Davis y Lin et al. (1989; 1997). El cuestionario se estructura en cuatro dimensiones: Utilidad percibida, Facilidad de uso, Facilidad de Aprendizaje, y Satisfacción; cada aspecto corresponde a una afirmación que se valora con una escala Likert de 1 al 4, siendo 1 'En desacuerdo' y 4 'De acuerdo'. Se incluyen, además, otras preguntas de tipo abierto para conocer la calificación de nivel de satisfacción, así como características que recomendaría agregar o mejorar del sistema, y cuál es el valor percibido del sistema para la administración de finca. El diligenciamiento del cuestionario fue en línea a través del propio Smartphone de cada participante, utilizando un formulario diseñado en Google Drive ${ }^{\circledR}$.

\section{Resultados}

\subsection{Resultados de la Fase de Desarrollo}

A partir de lo indicado en la Figura 1, el desarrollo del SIGF itagüe ${ }^{\circledR}$ implicó la ejecución de cuatro etapas centrales con sus respectivas actividades asociadas, como se detalla a continuación:

- Análisis de contexto de uso. En esta etapa se inició con la tarea de revisión de los propósitos y alcances del proyecto, para luego dar lugar a la creación del arquetipo (perfil) de los potenciales usuarios. Paralelamente se adelantaron las entrevistas con potenciales usuarios para aclarar las necesidades de uso, y se referenció en la literatura científica o empírica, otras soluciones SIGF similares disponibles en el mercado.

- Especificación de requisitos. En esta etapa se inició con la definición de la arquitectura general del SIGF y se continuó con el diseño de los mapas iniciales de navegación. Para tal fin, fue importante la determinación de roles en finca así como la especificación de tareas. 
- Producción de la solución (diseño del prototipo). Se partió de la creación de un prototipo de baja fidelidad (a partir de las funciones centrales), el cual fue mejorado y afinado a través de grupos focales, conformados por diseñadores y usuarios del SIGF. De esta manera se llegó al diseño gráfico y programación detallada del funcionamiento deseado en el SIGF, hasta consolidar un prototipo mínimo viable (PMV) y de alta fidelidad del sistema (ver Figura 3).

- Diseño de pruebas de usuario. Dado que la evaluación del SIGF se realizó mediante pruebas de usabilidad y de experiencia de usuario (UX User eXperience, en esta etapa se realize el diseño de las pruebas con base al test USE de Lund (2001).

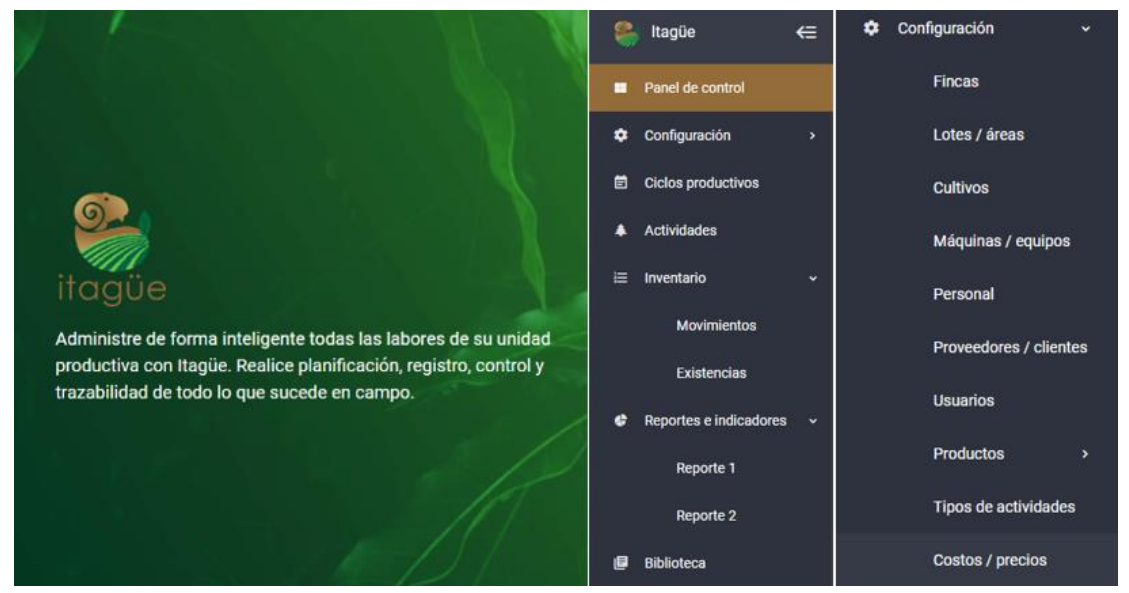

Fig. 3. Opciones de itagüe ${ }^{\circledR}$ versión producto mínimo viable

Fuente: http://itague.co

\subsection{Resultados de la Fase de Transferencia}

A partir del modelo propuesto por (Gonzalez, 2011), a continuación, se presentan los resultados del proceso de transferencia del SIGF itagüe $\AA$ :

- Actores. Los actores involucrados en el proceso fueron:

- Proveedor de la tecnología (suministrador o generador): Empresa pequeña, local y de base tecnológica, dedicada al desarrollo de software a la medida.

- Receptor de la tecnología (usuario o cliente): Propietarios de unidades productivas agrícolas vinculados a una Asociación de Productores de Fruta del sector hortofrutícola del Tolima, en Colombia. 
- Intermediario del proceso (acelerador, facilitador, dinamizador, asesor, difusor...): Grupo de investigación Universitario orientado a la gestión de la innovación y la competitividad empresarial.

- Modalidades. Las modalidades presentes en el proceso de creación del SIGF

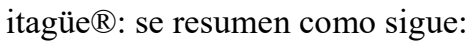

- Mecanismo de transferencia: El tipo de acuerdo entre el Proveedor y los Receptores (usuarios) inicialmente obedeció a cooperación técnica, sin costos de participación durante la fase piloto, con la posibilidad de que luego de un año de servicio, pudiese adquirirse como un servicio de base tecnológica incluido dentro del portafolio de productos y servicios del Proveedor.

- Vía de transferencia: El proceso de trabajo que dominó fue directo, es decir la comunicación directa entre el Proveedor y los Receptores (usuarios), a través de sesiones individuales y grupales programadas, además de visitas en campo.

- Formalidad de la transferencia. El acuerdo entre Proveedor y Receptores (usuarios), fue formal a través de un acuerdo escrito que gobernó los términos de la transferencia, en cuanto a entregables, contrapartidas, tiempos de respuesta y beneficios esperados.

- Enfoque de la transferencia: El enfoque dominante de transferencia fue de acceso a la tecnología por parte de los Receptores (usuarios), de esta.

- Ámbito y alcance de la transferencia: El ámbito geográfico considerado en la fase piloto del SIGF itagüe ${ }^{\circledR}$ fue regional, con alcance para productores de frutas de la región Tolima, proyectado para uso en otros sectores productivos a escala nacional e internacional.

- Contraprestación asociada a la transferencia. El acuerdo implicó una contraprestación económica y en especie hacia los productores, la primera para la adquisición de los equipos informáticos y la contratacción de conectividad en la unidad productive (finca), ambos requeridos para la transferencia. La segunda, para proporcionar el tiempo de personal experto en la producción agrícola en unidades productivas.

- Motivaciones. Las motivaciones, causas o factores que originaron, impulsaron y/o condicionan el desarrollo y la transferencia del SIGF itagüe ${ }^{\circledR}$ fueron:

- Las ventajas tecnológicas presentes en la zona, producto de la ampliación de la conectividad de las zonas rurales en Colombia, habilitando con ello, la posibilidad de integrar las TIC en esta actividad productiva.

- La actitud positiva y de superación de los Receptores (usuarios), para incursionar con éxito en el mercado internacional de frutas mediante certificaciones.

- La presencia de una convocatoria pública en Colombia a cargo del Ministerio de Agricultura y Desarrollo Rural (MADR), en alianza con la Red Avanza de 
Conocimiento RENATA, para financiar iniciativas que integraran la alianza entre empresas del sector TIC y las asociaciones consolidadas del sector Agrícola.

- Las presiones para alcanzar la rentabilidad que enfrentaban los productores de fruta de la Región Tolima, en tres aspectos en concreto: 1) la pérdida de valor comercial durante las épocas de cosecha por las leyes de la oferta y la demanda nacional 2) la falta de agroindustrias alternas que procesen los excedentes de producto, y 3) la persistencia de ineficiencias operativas y logísticas que les limita su capacidad para unirse a agro-cadenas de suministro de talla mundial.

- La experiencia del Proveedor en desarrollar soluciones tecnológicas basadas en la web, para el colectivo de productores hortofrutícolas de la región Tolima.

- Etapas. La transferencia de itagüe ${ }^{\circledR}$ como SIGF, implicó las siguientes etapas de trabajo:

- Identificación de la necesidad: La necesidad existente era la de contar con un SIGF, operando bajo tecnologías web, que estuviese adaptado para apoyar la gestión operativa de unidades productivas agrícolas, en las labores de planificación, programación, ejecución y control de tareas.

- Identificación de los actores. Se identificaron como actores centrales: a) el proveedor tecnológico: una empresa local perteneciente al Cluster TIC de la región Tolima, b) el receptor de la tecnología: una asociación productora de fruta en la región Tolima, c) el intermediario: un grupo de investigación vinculado a una Universidad privada que se localiza en la región Tolima.

- Negociación del acuerdo entre las partes. Los acuerdos entre las partes estuvieron determinados por los tiempos de ejecución, contrapartidas al presupuesto, compromisos de los beneficiarios receptores, compromisos de la empresa proveedora del sistema, entregables del proceso y estrategias de comunicación.

- Transferencia del sistema a los receptores. La transferencia se realizó a un grupo de Receptores, una vez estuvo desarrollado en su versión de Producto Mínimo Viable (PMV). Los resultados de la transferencia se vieron reflejados en el afinamiento del itagüe ${ }^{\circledR}$ en lo relativo a generar condiciones de diseño para proveer mayor a utilidad de la herramienta y mayor facilidad de aprendizaje, y uso para el usuario final.

\subsection{Resultado Fase de Evaluación de Resultados}

A efectos de realizar los análisis de resultados en el área de experiencia de usuario, se tomaron como base las recomendaciones de Tullis y Albert (2013) y Hartson y Pyla (2012), quienes sugieren que con el uso de muestras pequeñas de 8 a 10 participantes se logran 
Integración de sistemas de información para la innovación en la gestión operativa de unidades productivas agrícolas. Un caso de estudio en Colombia

resultados significativos. En el caso del proyecto itagüe ${ }^{\circledR}$, se utilizaron las dimensiones y preguntas presentadas en la Tabla 1. Se verificó la fiabilidad del instrumento con el Alpha de Cronbach $(\alpha)$, y los resultados fueron significativos en cada dimensión: Utilidad, $\alpha=$ .962 ( 8 elementos); Facilidad de uso, $\alpha=.941$ ( 9 elementos); Facilidad de aprendizaje, $\alpha=$ .889 (3 elementos); y Satisfacción, $\alpha=.877$ ( 5 elementos).

Tabla 1. Variables del instrumento aplicado para evaluación de la experiencia de usuario

\begin{tabular}{|c|c|c|c|}
\hline Dimensión & Preguntas & Escala & Tipo \\
\hline \multirow{8}{*}{$\begin{array}{l}\text { Utilidad } \\
\text { (UT) }\end{array}$} & - Me ayudará a ser más productivo en la administración de la finca & \multirow{8}{*}{$\begin{array}{c}\text { Escala Likert } \\
{[1} \\
\text { Desacuerdo... } \\
4 \text { Acuerdo] }\end{array}$} & \multirow[t]{8}{*}{ Ordinal } \\
\hline & - Me dará más control sobre la planeación de las actividades de finca & & \\
\hline & - Hará los procesos de control de la finca más fáciles & & \\
\hline & - Me ahorrará tiempo en el proceso de administración de la finca & & \\
\hline & - $\quad$ Responde a mis necesidades de administración de la finca & & \\
\hline & - $\quad$ Responde a mis necesidades de registro de administración de finca & & \\
\hline & - $\quad$ Responde a mis necesidades de control de administración de la finca & & \\
\hline & $\begin{array}{l}\text { - Responde a mis necesidades de trazabilidad de administración en } \\
\text { finca }\end{array}$ & & \\
\hline \multirow{9}{*}{$\begin{array}{l}\text { Facilidad } \\
\text { de Uso } \\
\text { (FU) }\end{array}$} & - Logré hacer sin mayor dificultad los ejercicios que me solicitaron & \multirow{9}{*}{$\begin{array}{c}\text { Escala Likert } \\
\qquad 1 \\
\text { Desacuerdo... } \\
4 \text { Acuerdo] }\end{array}$} & \multirow[t]{9}{*}{ Ordinal } \\
\hline & - Me siento capaz de usarlo sin que alguien más me explique cómo & & \\
\hline & - $\quad$ Considero que el número de pasos para una tarea es el adecuado & & \\
\hline & - $\quad$ El fácil ingresar información personalizada de mi finca & & \\
\hline & - $\quad$ El diseño visual del sistema es agradable & & \\
\hline & - $\quad$ Puedo usar el sistema sin consultar las opciones de ayuda & & \\
\hline & - Al personal de la finca le gustaría usar el sistema & & \\
\hline & - $\quad$ Si me llegaba a equivocar, fui capaz de recuperarme de los errores & & \\
\hline & - $\quad$ Puedo guiarme sin problemas con los iconos (dibujos de los botones) & & \\
\hline \multirow{3}{*}{$\begin{array}{c}\text { Facilidad } \\
\text { de } \\
\text { Aprendizaje } \\
\text { (FA) }\end{array}$} & - $\quad$ Soy capaz de hacer uso del sistema sin la ayuda de otra persona & \multirow{3}{*}{$\begin{array}{c}\text { Escala Likert } \\
\qquad 1 \\
\text { Desacuerdo... } \\
4 \text { Acuerdo] }\end{array}$} & \multirow[t]{3}{*}{ Ordinal } \\
\hline & - Después de terminado el curso, me siento capaz de recordar su uso & & \\
\hline & - $\quad$ Itagüe es fácil de aprender a usarlo & & \\
\hline \multirow{5}{*}{$\begin{array}{l}\text { Satisfacción } \\
\qquad(\mathrm{ST})\end{array}$} & - $\quad$ Estoy satisfecho con el sistema Itagüe & \multirow{5}{*}{$\begin{array}{c}\text { Escala Likert } \\
\text { [1 } \\
\text { Desacuerdo... } \\
4 \text { Acuerdo] }\end{array}$} & \multirow[t]{5}{*}{ Ordinal } \\
\hline & - $\quad$ Recomendaría Itagüe a un amigo administrador de otra finca & & \\
\hline & - $\quad$ Itagüe funcionó de la forma en que lo había imaginado & & \\
\hline & - $\quad$ Tiene previsto utilizar Itagüe en su finca & & \\
\hline & - Itagüe es un sistema excelente & & \\
\hline
\end{tabular}

Fuente: Este estudio a partir de la adaptación del Test USE (Gao et al., 2018; Lund, 2001). 
A efectos de analizar los resultados y para reducir la dimensión de las variables originales y ganar con ello capacidad discriminante y de explicación se realizó un análisis factorial y se crearon dos nuevas variables (ver Tabla 2).

Tabla 2. Nuevas variables estimadas a partir de análisis factorial

\begin{tabular}{c|c|c|c}
\hline Nuevas variables & Método & Escala/valor & Tipo \\
\hline Categoría de edad & Re-categorización según respuesta inicial & $\begin{array}{c}\leq 55 \text { años } \\
>55 \text { años }\end{array}$ & Ordinal \\
\hline Nivel de dominio de TI & $\begin{array}{c}\text { Suma aritmética de las puntuaciones de las } \\
\text { cinco preguntas realizadas) }\end{array}$ & $\begin{array}{c}\text { Bajo } \\
\text { Intermedio }\end{array}$ & Ordinal \\
\hline
\end{tabular}

Para el análisis integral de las dimensiones usadas para valoración de itagüe ${ }^{\circledR}$ por parte de los usuarios, se tuvieron en cuenta las nuevas variables estimadas Categoría de edad y Nivel de integración TI (Tabla 2), que en su conjunto revelan el valor promedio en la puntuación no normalizada del primer componente principal extraído, y que varían en algún grado por razón de la edad y/o el nivel de uso de las TI (ver figura 4).
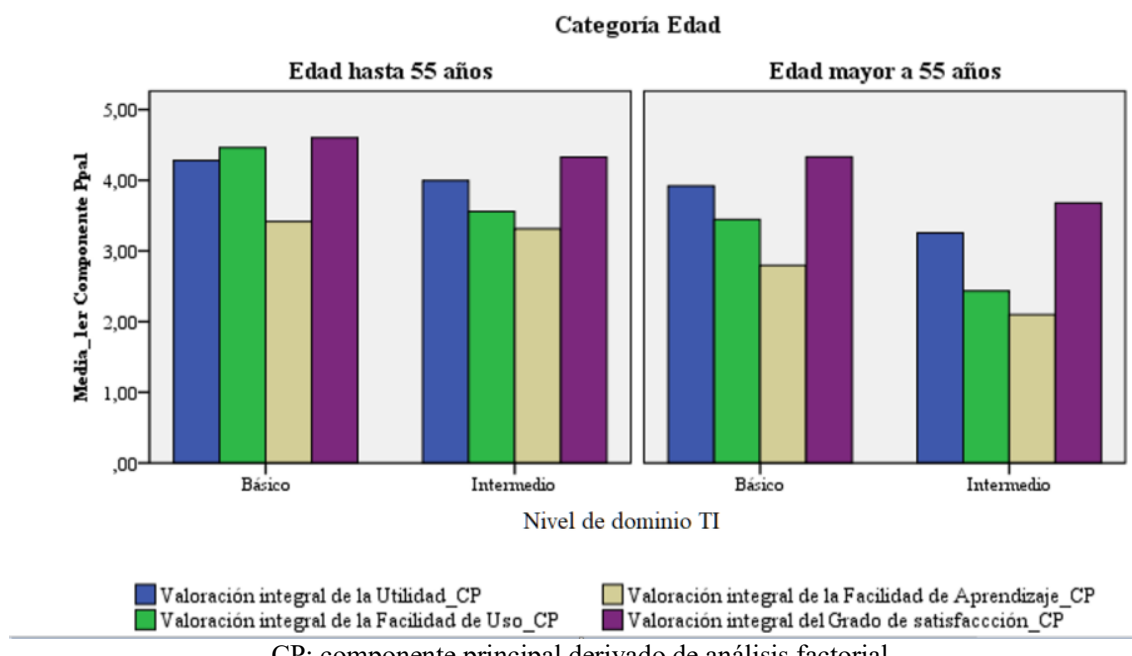

$\mathrm{CP}$ : componente principal derivado de análisis factorial

Fig.4. Puntuaciones promedio de la valoración integral de cada dimensión del test USE

La comparación estadística de las puntuaciones dadas a cada dimensión evaluada a través del test no paramétrico para muestras independientes (Test $U$ de Mann-Whitney) evidencian que la valoración dada a itagüe ${ }^{\circledR}$ en las cuatro dimensiones que contempla el test USE, fue significativamente diferente en lo relativo a Facilidad de uso, siendo superiores en promedio las calificaciones dadas por las personas no mayores a 55 años y con nivel de básico en el dominio de TI (Tabla 3). 
Integración de sistemas de información para la innovación en la gestión operativa de unidades productivas agrícolas. Un caso de estudio en Colombia

Tabla 3. Comparación de respuestas entre grupos - Test estadístico $U$

\begin{tabular}{|c|c|c|c|c|}
\hline \multirow[t]{2}{*}{ Componente } & \multicolumn{2}{|c|}{ Edad del usuario } & \multicolumn{2}{|c|}{ Nivel de dominio de TI } \\
\hline & P-Valor & Sig. Test & P-Valor & Sig. Test \\
\hline $\begin{array}{l}\text { La valoración de Utilidad_CP, es la misma } \\
\text { entre categorías }\end{array}$ & 149,000 & Retiene $\mathrm{H}_{\mathrm{o}}$ & 240,000 & Retiene $\mathrm{H}_{\mathrm{o}}$ \\
\hline $\begin{array}{l}\text { La valoración de Facilidad de Uso_CP, es la } \\
\text { misma entre categorías }\end{array}$ & 48,000 & Rechaza $\mathbf{H}_{0}$ & 41,000 & Rechaza $\mathbf{H}_{0}$ \\
\hline $\begin{array}{l}\text { La valoración Facilidad de Aprendizaje_CP, } \\
\text { es la misma entre categorías }\end{array}$ & 73,000 & Retiene $\mathrm{H}_{\mathrm{o}}$ & 310,000 & Retiene $\mathrm{H}_{\mathrm{o}}$ \\
\hline $\begin{array}{l}\text { La valoración de Satisfacción_CP, es la } \\
\text { misma entre categorías }\end{array}$ & 268,000 & Retiene $\mathrm{H}_{\mathrm{o}}$ & 132,000 & Retiene $\mathrm{H}_{\mathrm{o}}$ \\
\hline
\end{tabular}

\subsection{Discusión de resultados}

Los resultados obtenidos evidencian dos logros en particular: el primero referente al desarrollo de una herramienta innovadora que demostró ser útil para transformar la gestión operativa de unidades productivas agrícolas, específicamente en la planificación, programación, ejecución y control de tareas de productores del sector hortofrutícola. El segundo se constituye con el involucramiento de usuarios con experiencia en la actividad agrícola y conocimientos básicos en el uso de TI, quienes contribuyeron de manera significativa en las etapas de pruebas y afinamiento del SIFG desarrollado, para hacer de itagüe ${ }^{\circledR}$ un sistema que incorporara en su funcionamiento y contenidos, tanto la experiencia del productor agrícola como la del desarrollador del sistema.

En lo referente a la incorporación de las TIC y la innovación empresarial en el sector agrícola, sin lugar a dudas, un componente clave a considerar es la edad del productor adoptante. Estudios como el adelantado por Lewis en 1998, evidenció que los agricultores de más edad no usan tantas fuentes de información como sus colegas más jóvenes, puesto que confían más en su experiencia. Por el contrario, los jóvenes cada vez más apoyan sus decisiones utilizando herramientas TIC como Internet, computador portátil, tableta, smartphone y correo electrónico (Sonderegger et al., 2016). En el caso de itagüe ${ }^{\circ}$, dicho patrón fue consecuente con lo indicado por Lewis (1998), así, los productores de mayor edad se mostraron más confiados en su propia experiencia, y fueron en un principio, más escépticos sobre la verdadera conveniencia de la herramienta, no obstante, al mismo tiempo, presentaron una actitud más favorable que los jóvenes hacia la incorporación de la nueva solución diseñada, en tanto reconocieron luego de la orientación técnica recibida, su gran potencial para transformar y hacer más eficiente la gestión operativa de las fincas, esto es, los agricultores de mayor edad y más experimentados, dada su experiencia y conocimiento, suelen tienen menos demanda de información de gestión, lo que puede dar como resultado una menor demanda de tecnología (computadores) y con un dominio básico de ésta en comparación con sus colegas más jóvenes, hecho que se alinea con los hallazgos de Carrer et al. (2017) quien evidenció que los agricultores con un mayor experiencia si 
bien tienen más probabilidades de realizar inversiones, a la vez tienden a sobreestimar los resultados esperados de sus decisiones, factores que a su vez, pueden incidir en el incremento de la probabilidad de adopción y uso de nuevas tecnologías de gestión agrícola.

Respecto al uso de la asistencia técnica, en el caso de itagüe ${ }^{\circledR}$ se eliminaron barreras en la comunicación y lo cual ejerció un efecto positivo en la adopción e intensidad en el uso la herramienta por parte del grupo de usuarios de mayor edad, participante en el grupo piloto de validación; los resultados positivos corresponden con las recomendaciones dadas en el estudio de Morris, Henley, y Dowell (2017) y los hallazgos del trabajo de Carrer et al. (2017).

\section{Conclusiones}

La modernización de cualquier sistema de producción agrícola demanda la integración de las TIC a nivel operativo y gerencial para lograr niveles de productividad y rentabilidad que sean competitivos en dicho sector. El caso analizado de itagüe ${ }^{\circledR}$ en la región Tolima (Colombia), demuestra que los SIGF apoyados en ambientes web constituyen una herramienta viable para modernizar e innovar en la gestión operativa de unidades productivas agrícolas.

El estudio del proceso de desarrollo y transferencia del SIGF itagüe ${ }^{\circledR}$ provee aportaciones desde los puntos de vista metodológico y práctico. En primer lugar, las metodologías utilizadas para diseñar y desarrollar itagüe ${ }^{\circledR}$, confirman la conveniencia de utilizar metodologías, técnicas y herramientas de diseño centrado en el usuario, para asegurar la eficacia, eficiencia y satisfacción con la que el producto final alcance y satisfaga los objetivos específicos de sus usuarios finales tanto para aquellos con dominio básico en el uso de tecnologías como con los que tienen un nivel intermedio. Desde el punto de vista práctico, los resultados obtenidos con itagüe ${ }^{\circledR}$ evidencian que para logar una exitosa transferencia de un SIGF es importante involucrar a los usuarios finales (productores agrícolas), desde las etapas iniciales del proceso de desarrollo hasta la etapa de configuración final así como su respectiva transferencia y evaluación, tanto a los más experimentados en las prácticas agrícolas como a los más jóvenes.

De cara a proyectos futuros itagüe ${ }^{\circledR}$ representa la oportunidad para demostrar que un SIGF puede representar una herramienta para mejorar la eficiencia de la gestión de fincas hortofrutícolas, con la posibilidad de que se puedan transferir para otro tipo de producción agrícola, asimismo, demuestra la importancia de incorporar en los procesos de diseño, Desarrollo, transferencia y evaluación de este tipo de sistemas la experiencia de los propios usuarios finales. 
Integración de sistemas de información para la innovación en la gestión operativa de unidades productivas agrícolas. Un caso de estudio en Colombia

\section{Agradecimientos}

Las autoras agradecen el apoyo financiero provisto por la Convocatoria pública número 02 de 2017 de la alianza MINTIC-RENATA para el desarrollo del Proyecto 'Planntic: Sistema de gestión y trazabilidad de operaciones en unidades productivas agrícolas'. De igual manera agradecen los aportes del equipo técnico organizado para la ejecución del Proyecto por parte de la empresa TIC MAKERS S.A.S Colombia, especialmente a David Castiblanco por su valioso rol como líder de proyecto, así como a los beneficiarios del Proyecto y miembros de la Cooperativa Multiactiva de Manguicultores de Colombia Promango (Tolima - Colombia).

\section{Referencias}

Bilali, E. H., \& Allahyari, M. S. (2018). Transition towards sustainability in agriculture and food systems: Role of information and communication technologies. Information Processing in Agriculture, 5(4), 456-464. https://doi.org/10.1016/j.inpa.2018.06.006

Carrer, M. J., de Souza Filho, H. M., \& Batalha, M. O. (2017). Factors influencing the adoption of Farm Management Information Systems (FMIS) by Brazilian citrus farmers. Computers and Electronics in Agriculture, 138, 11-19. https://doi.org/10.1016/j.compag.2017.04.004

Davis, F. (1989). Perceived usefulness, perceived ease of use, and user acceptance of information technology. MIS Quarterly, 13(3), 319-340.

Fountas, S., Carli, G., Sorensen, C. G., Tsiropoulos, Z., Cavalaris, C., Vatsanidou, A., Liakos, B., Canavari, M., Wiebensohn, J., \& Tisserye, B. (2015). Farm management information systems: Current situation and future perspectives. Computer and Electronics in Agriculture, 115, 40-50.

Gao, M., Kortum, P., \& Oswald, F. (2018). Psychometric evaluation of the USE (usefulness, satisfaction, and ease of use) questionnaire for reliability and validity. Proceedings of the Human Factors and Ergonomics Society, 3, 1414-1418. https://doi.org/10.1177/1541931218621322

Gonzalez, J. (2011). Manual transferencia de tecnología y conocimiento. The transfer institute. http://www.negociotecnologico.com

Hartson, R., \& Pyla, P. (2012). The UX Book: Process and Guidelines for Ensuring a Quality User Experience. Morgan Kaufmann.

Kaloxylos, A., Eigenmann, R., Teye, F., Politopoulou, Z., Wolfert, S., Shrank, C., Dillinger, M., Lampropoulou, I., Antoniou, E., Pesonen, L., Nicole, H., Thomas, F., Alonistioti, N., \& Kormentzas, G. (2012). Farm management systems and the Future Internet era. Computers and Electronics in Agriculture, 89, 130-144. https://doi.org/10.1016/j.compag.2012.09.002

Lin, H., Choong, Y.-Y., \& Salvendy, G. (1997). A proposed index of usability: a method for comparing the relative usability of different software systems. Behaviour and information technology, 4/5, 267-278. http://dx.doi.org/10.1080/014492997119833 
Lund, A. (2001). Measuring usability with the USE questionnaire. Usability and user experience special interest group, $8(2)$. https://www.researchgate.net/publication/230786746_Measuring_Usability_with_the_USE_Ques tionnaire

Morris, W., Henley, A., \& Dowell, D. (2017). Farm diversification, entrepreneurship and technology adoption: Analysis of upland farmers in Wales. Journal of Rural Studies, 53, 132-143. https://doi.org/10.1016/j.jrurstud.2017.05.014

OCDE. (2015). Revisión de la OCDE de las políticas Agrícolas: Colombia 2015. Revisiones sobre politicas agricolas, 27.

Pradhan, R. P., Mallik, G., \& Bagchi, T. P. (2018). Information communication technology (ICT) infrastructure and economic growth: A causality evinced by cross-country panel data. IIMB Management Review, 30(1), 91-103. https://doi.org/10.1016/j.iimb.2018.01.001

Sánchez, D., Ibarra, J., Flores, B., López, G. (2012). Adopción del Estándar ISO 9241-210:2010 en la Construcción de Sistemas Interactivos Basados en Computadora. Congreso Internacional de Investigación e Innovación en Ingeniería de Software - CONISOFT2012.

Sonderegger, A., Schmutz, S., \& Sauer, J. (2016). The influence of age in usability testing. Applied Ergonomics, 52, 291-300. https://doi.org/10.1016/j.apergo.2015.06.012

Tullis, T., \& Albert, B. (2013). Measuring user experience. Collecting, analyzing, and presenting usabilidty metrics (2nd ed.). Morgan Kaufmann Publishers Inc.

Tummers, J., Kassahun, A., \& Tekinerdogan, B. (2019). Obstacles and Features of Farm Management Information Systems: A Systematic Literature Review. Manuscript in preparation, 157(December 2018), 189-204. https://doi.org/10.1016/j.compag.2018.12.044 